\title{
Study of three-dimensional NMR forward modeling using finite element method
}

\author{
Zhiping REN ${ }^{1,2, a^{*}}$, Xiu LI ${ }^{2, b}$ and $Y u L^{2, c}$ \\ ${ }^{1}$ School of Geology Engineering and Geomatics, Chang'an University, Xi'an, Shaanxi, China \\ ${ }^{2}$ School of Electronic Engineering, Xi'an Shiyou University, Xi'an, Shaanxi, China \\ arenzp@xsyu.edu.cn, ${ }^{\mathrm{b}}$ lixiu@chd.edu.cn, ${ }^{\mathrm{c}}$ leiyu5626633 @qq.com
}

\begin{abstract}
Keywords: NMR; three-dimensional; forward modeling; finite element
Abstract. In order to improve the accuracy of groundwater detection, the forward problem of NMR response in three-dimensional is discussed in this paper. The three-dimensional magnetic field equations and boundary conditions in frequency domain with the loop source are derived by Maxwell's equations and then the functional expression is given. The finite element method is applied to calculate the forward modeling. Numerical simulation shows that the excitation pulse moment is help to increase the detecting depth. Shallow aquifer resistivity has more influence on the initial signal amplitude, the higher of the resistivity, the smaller of the maximum of the initial amplitude will be. The research will provide the necessary reference for complex structure groundwater investigation with NMR.
\end{abstract}

\section{Introduction}

Water is one of the most important resources for human survival and development. Geophysical methods have been widely applied to explore groundwater, such as induced polarization, apparent resistivity and transient electromagnetic method et al. Most of the methods detect the aquifer indirectly by comparing the differences of water structure and it is difficult to accurately determine the rock water contents.

Comparing with the traditional geophysical methods, Nuclear Magnetic Resonance (NMR) has the advantages of high resolution, high efficiency and the uniqueness of solution et al. NMR for surface prospecting of hydrocarbons was proposed by Varian in $1962^{[1]}$. This method has been tested in many countries, like Russian, France, US and Germany et $\mathrm{al}^{[2,3,4]}$. In China, many scholars have carried out a series of work on NMR theory, coil design and instrument development, which greatly promoted the development of NMR technology ${ }^{[5,6,7,8]}$. At present, many studies are based on the forward and inversion modeling with one dimensional, which have been developed maturely. While the storage structures of actual underground water is complex and it is necessary to study the NMR detection with three dimensional.

\section{Theoretical analysis of NMR}

NMR is a phenomenon that produced by the interaction between nuclear magnetism and external electromagnetic field. When an external electromagnetic field is applied to the natural geomagnetic field, its spin system will be polarized and the magnetic moments will process from their equilibrium with Larmor frequency. If another alternating magnetic field with the same frequency is applied in the vertical direction of static magnetic field, the nucleus in low energy absorb the transmit electromagnetic energy and transit to a higher energy level.

Surface NMR method regards the natural geomagnetic field as the static magnetic field. The external electromagnetic field is created with the help of an induction coil on the ground. The induced current in the coil carries the same frequency as the Larmor precession frequency of protons to make the macroscopic magnetic moments precessing from their equilibrium. When this field is terminated, they return to their initial position and generate a magnetic field with initial signal amplitude 


$$
E_{0}(q)=\frac{\omega_{0} M_{0}}{I} \int_{V} B_{\perp} \sin \left(\gamma \frac{B_{\perp}}{I} q\right) n(r) d V
$$

Where, $\omega_{0}$ is the Larmor frequency, $M_{0}$ is the macroscopic magnetic moment, $I$ is the emission current, $B_{\perp}$ is the perpendicular component to natural geomagnetic field of excitation field normal, $n$ is the water content, $\gamma$ is the gyromagnetic ratio, $q$ is the pulse moment.

\section{NMR forward modeling}

By Fourier transform of Maxwell equation in time domain, then

$$
\left\{\begin{array}{l}
\nabla \times \boldsymbol{E}=i \omega \mu \boldsymbol{H} \\
\nabla \times \boldsymbol{H}=(\sigma-i \mu \varepsilon) \boldsymbol{E}+\boldsymbol{J}_{s}
\end{array}\right.
$$

Where $\boldsymbol{E}$ and $\boldsymbol{H}$ are the electric and magnetic field vector, $\omega$ is the angular frequency of the loop source, $\varepsilon, \mu, \sigma$ are the permittivity, magnetic permeability and electrical conductivity respectively, $\boldsymbol{J}_{s}$ is the current density of the external current source. Taking the curl on both sides of formula (2), then

$$
\nabla \times \nabla \times \boldsymbol{H}-k^{2} \boldsymbol{H}=\nabla \times \boldsymbol{J}
$$

Where $k^{2}=\omega^{2} \varepsilon \mu+i \omega \mu \sigma$. Due to the absence of magnetic charge and current density on the surface, the normal and tangential continuity in the internal electrical interface, the boundary conditions of equation (3) is given by the following

$$
\boldsymbol{n} \times(\nabla \times \boldsymbol{H})+\gamma_{h} \boldsymbol{n} \times(\boldsymbol{n} \times \boldsymbol{H})=\boldsymbol{V}
$$

Where, $\boldsymbol{n}$ is the normal unit vector of $S_{2}$, and $V, \gamma_{h}$ is the known quantities of the boundary conditions. In order to calculate equation (3) with boundary condition (4), the following function is introduced according to the variational principle

$$
\boldsymbol{F}(\boldsymbol{H})=\frac{1}{2} \iiint_{V}\left[(\nabla \times \boldsymbol{H}) \cdot(\nabla \times \boldsymbol{H})-k^{2} \boldsymbol{H} \cdot \boldsymbol{H}\right] d V-\iiint_{V} \boldsymbol{H} \cdot\left(\nabla \times \boldsymbol{J}_{s}\right) d V
$$

The magnetic field is calculated by the finite element method. Divided the volume into small units, and denote $\boldsymbol{R}=\nabla \times \boldsymbol{J}_{s}$. Assuming the uniformity in the unit internal, then the problem of solving stagnation point of (5) is equivalent to solve the system of equations

$$
\left\{\begin{array}{l}
\frac{\partial F\left(H_{x}\right)}{\partial H_{x i}}=\sum_{e=1}^{n} \frac{\partial F_{e}}{\partial H_{x i}^{e}}=0 \\
\frac{\partial F\left(H_{y}\right)}{\partial H_{y i}}=\sum_{e=1}^{n} \frac{\partial F_{e}}{\partial H_{y i}^{e}}=0 \\
\frac{\partial F\left(H_{z}\right)}{\partial H_{z i}}=\sum_{e=1}^{n} \frac{\partial F_{e}}{\partial H_{z i}^{e}}=0
\end{array}\right.
$$

Where $n$ is the number of units, $e$ is the unit serial number, $H_{x i}, H_{y i}, H_{z i}$ is the magnetic components of unite node $i$, then

$$
\left\{\begin{array}{l}
\frac{\partial F_{e}}{\partial H_{x i}^{e}}=\sum_{j=1}^{n e} \iiint_{e}\left[\left(\frac{\partial N_{i}^{e}}{\partial y} \frac{\partial N_{j}^{e}}{\partial y}+\frac{\partial N_{i}^{e}}{\partial z} \frac{\partial N_{j}^{e}}{\partial z}-k^{2} N_{i}^{e} N_{j}^{e}\right) H_{x j}^{e}-\frac{\partial N_{i}^{e}}{\partial y} \frac{\partial N_{j}^{e}}{\partial x} H_{y j}^{e}-\frac{\partial N_{i}^{e}}{\partial z} \frac{\partial N_{j}^{e}}{\partial x} H_{z j}^{e}\right] d x d y d z=\sum_{j=1}^{n e} \iiint_{e} N_{i} N_{j} d x d y d z \cdot R_{x j} \\
\frac{\partial F_{e}}{\partial H_{y i}^{e}}=\sum_{j=1}^{n e} \iiint_{e}\left[-\frac{\partial N_{i}^{e}}{\partial x} \frac{\partial N_{j}^{e}}{\partial y} H_{x j}^{e}+\left(\frac{\partial N_{i}^{e}}{\partial x} \frac{\partial N_{j}^{e}}{\partial x}+\frac{\partial N_{i}^{e}}{\partial z} \frac{\partial N_{j}^{e}}{\partial z}-k^{2} N_{i}^{e} N_{j}^{e}\right) H_{y j}^{e}-\frac{\partial N_{i}^{e}}{\partial z} \frac{\partial N_{j}^{e}}{\partial y} H_{z j}^{e}\right] d x d y d z=\sum_{j=1}^{n e} \iiint_{e} N_{i} N_{j} d x d y d z \cdot R_{y j} \\
\frac{\partial F_{e}}{\partial H_{z i}^{e}}=\sum_{j=1}^{n e} \iiint_{e}\left[-\frac{\partial N_{i}^{e}}{\partial x} \frac{\partial N_{j}^{e}}{\partial z} H_{x j}^{e}-\frac{\partial N_{i}^{e}}{\partial y} \frac{\partial N_{j}^{e}}{\partial z} H_{y j}^{e}+\left(\frac{\partial N_{i}^{e}}{\partial x} \frac{\partial N_{j}^{e}}{\partial x}+\frac{\partial N_{i}^{e}}{\partial y} \frac{\partial N_{j}^{e}}{\partial y}-k^{2} N_{i}^{e} N_{j}^{e}\right) H_{z j}^{e}\right] d x d y d z=\sum_{j=1}^{n e} \iiint_{e} N_{i} N_{j} d x d y d z \cdot R_{z j}
\end{array}\right.
$$

Where $N_{i}^{e}$ is the shape function for node $i, n e$ is the number of nodes for the unit $e$. Combing the conditions of all stagnation points and then the magnetic field intensity of arbitrary points in the space can be obtained by solving the following function. 


$$
\left|\begin{array}{lll}
K_{x x} & K_{x y} & K_{x z} \\
K_{y x} & K_{y y} & K_{y z} \\
K_{z x} & K_{z y} & K_{z z}
\end{array}\right| \begin{aligned}
& H_{x} \\
& H_{y} \\
& H_{z}
\end{aligned}|=| N_{i j}|| \begin{aligned}
& R_{x} \\
& R_{y} \\
& R_{z}
\end{aligned} \mid
$$

\section{Effects of aquifer parameters on NMR response}

In order to obtain the NMR response characteristics of aquifer with three-dimensional, the numerical simulation of NMR response with different aquifer depth and variety of resistivity is studied in the following. The designed model is illustrated in Fig 1, which contain a cube aquifer with size $10 m * 10 m * 1 m$ in the homogeneous medium with resistivity $100 \Omega \cdot m$.

The boundary location of the cube is assumed as $x:-5 m \sim 5 m, y:-5 m \sim 5 m$ and the center of the aquifer is located in $z$ axis. Therefore the coordinates of the measuring points are(1) $(-2.5,-2.5,0)$, (2) $(-2.5,0,0)$, (3) $(-2.5,2.5,0)$, (4) $(0,-2.5,0)$, (5) $(0,0,0)$, (6) $(0,2.5,0)$, (7) $(2.5,-2.5,0)$, (8) $(2.5,0,0)$, (9) $(2.5,2.5,0)$ with the unit as meter. During the numerical simulation, the terrestrial magnetism is assumed as $48000 \mathrm{nT}$, geomagnetic inclination as $60^{\circ}$, geomagnetic declination as $0^{\circ}$, and the Lamor frequency is $2350 \mathrm{~Hz}$.

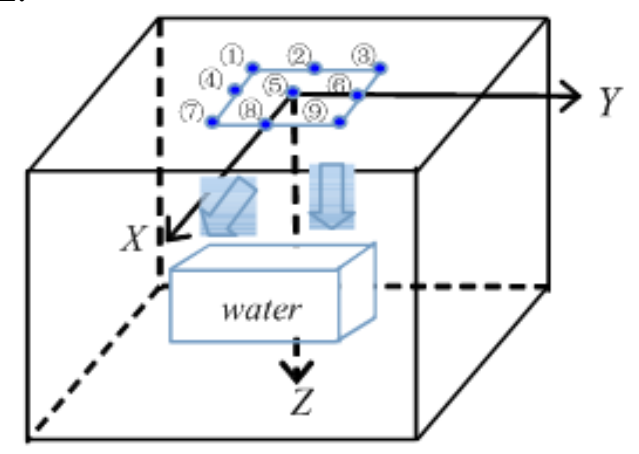

Fig.1 the mode of aquifer

\section{Effects of aquifer depth on NMR response}

The aquifer resistivity is set to $5 \Omega \cdot m$, when the aquifer depth are taken as $6 \mathrm{~m}, 9 \mathrm{~m}, 12 \mathrm{~m}$, the relationships between pulse moment $q$ and initial amplitude $E_{0}$ are illustrated in Fig 2. It demonstrates that the deeper the depth of aquifer, the smaller the response amplitude of measuring points will be, and the lower resolution is, while the pulse moment corresponding to the maximum initial amplitude becomes larger. Therefore, the NMR signal can reflect the variation of aquifer depth. Increasing the pulse moment of emission signal can expand the exploration depth and acquire the more information about the deeper aquifer.

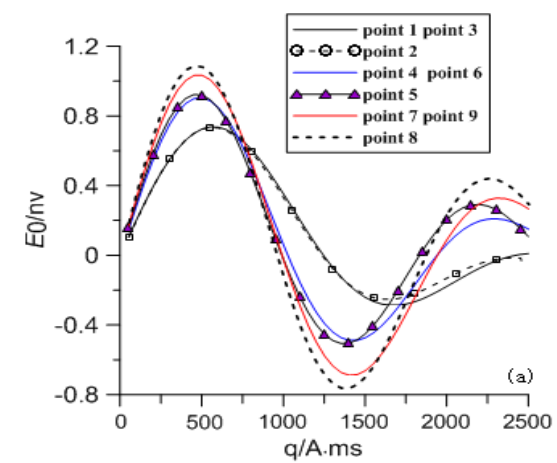

(a) The depth of the center is $6 \mathrm{~m}$

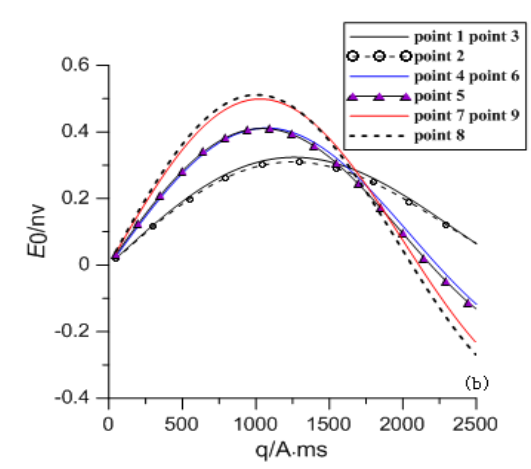

(b) The depth of the center is $9 \mathrm{~m}$ $12 \mathrm{~m}$

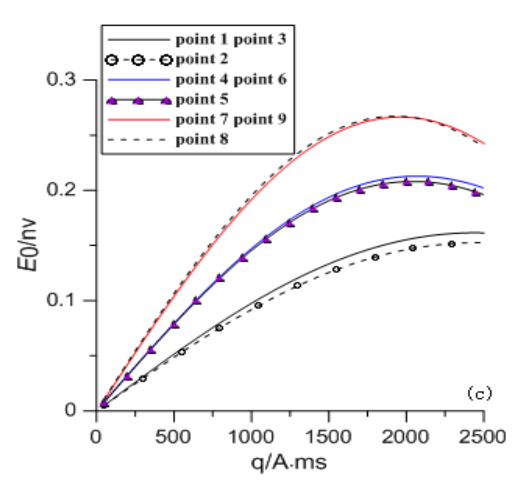

(c) The depth of the center is

Fig. 2 The effect of aquifer depth on NMR response 


\section{Effects of aquifer resistivity on NMR response}

Fig 3 illustrates the influences of aquifer resistivity to the initial amplitude with the condition that the central position of the aquifer is $(0,0,6)$ and the resistivity are $50 \Omega \cdot m, 100 \Omega \cdot m, 200 \Omega \cdot m$ respectively.

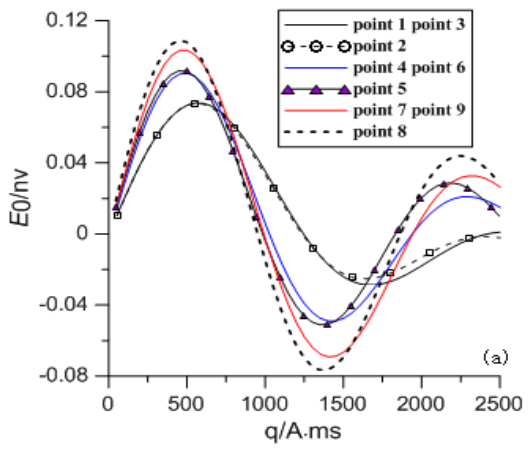

(a) The aquifer resistivity is $50 \Omega \cdot m$ is $200 \Omega \cdot m$

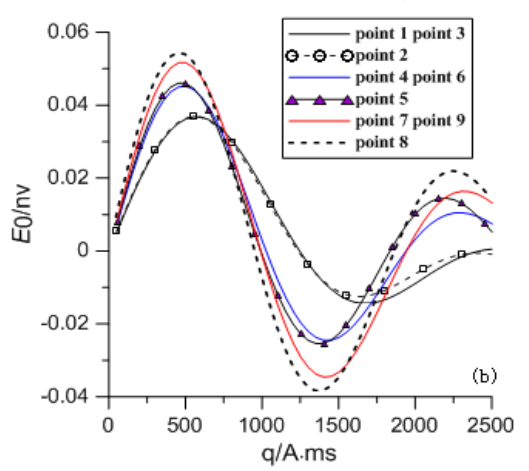

(b) The aquifer resistivity is $100 \Omega \cdot m$

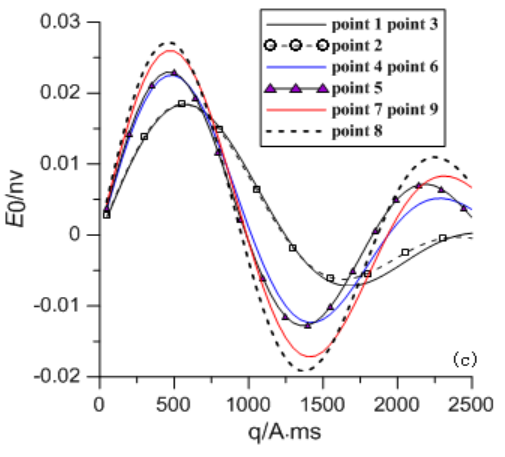

(c) The aquifer resistivity

Fig. 3 The effect of aquifer resistivity on NMR response

Fig 3 show that aquifer resistivity change has less effect on the curve shapes for different measuring points. The relationship between initial amplitude and pulse is remaining the same, while the amplitude of the curve is sensitive to resistivity change. With the same pulse moment, the larger aquifer resistivity, the smaller response amplitude is. The approximate linear relationship between aquifer resistivity and maximum initial amplitude provides the theoretical basis for the inversion calculation of shallow aquifer.

\section{Summary}

Excitation magnetic field distribution in frequency domain is essential to calculate three-dimensional magnetic resonance response. From the variational problem of harmonic magnetic field, the finite element equations are obtained by solving the stagnation. The relationship between NMR response characteristics and the parameters of water structure are studied in this paper. The results show that the NMR resonance is sensitive to the variations of water depth. The NMR detection depth is mainly affected by the impulse moment. Shallow aquifer resistivity has more influence on the initial signal amplitude, the higher of the resistivity, the smaller of the maximum of the initial amplitude will be. The result has important reference value to study the resistivity inversion calculation.

\section{ACKNOWLEDGEMENTS}

This research is supported by the National Natural Science Foundation of China (No. 51139004), Shaanxi Training Programs of Innovation and Entrepreneurship for Undergraduates (1364).

\section{References}

[1] Y. Pan, C. Zhang. New Sphere of Nuclear Magnetic Resonance Technology Application .CT Theory and Applications. 8(1998) 5-8.

[2] Z. Kimmie. The underground application of magnetic resonance soundings. Journal of Applied Geophysics, 75(2011) 220-226.

[3] A. Legchenko, J. Vouillamoz, J. Roy. Application of the magnetic resonance sounding method to the investigation of aquifers in the presence of magnetic materials. Geophysics, 6(2010): L91-L100.

[4] U. Yaramanci, G. Lange, M. Hetrich, Aquifer characterisation using surface NMR jointly with other geophysical techniques at the Nauen/Berlin test site. Journal of Applied Geophysics, 50 (2002): $47-65$. 
[5] R. Zhang, X. Hu, D. Yang, et al. Review of development of surface nuclear magnetic resonance. Progress in Geophysics, 1(2006): 285-289.

[6] Z. Li, Y. Pan, B. Zhang, et al. Using NMR method research the hydrogeology problems and practical examples. Hydrogeology and Engineering Geology, 4(2003): 50-54.

[7] J. Lin, C. Jiang, Q. Duan. The situation and progress of magnetic resonance sounding for groundwater investigations and underground application. Journal of Jilin University (Earth Science Edition), 5(2012): 1560-1570.

[8] A. Weng, Z. Li, X. Wang. A study on surface nuclear magnetic resonance over layered conductive earth. Chinese Journal of Geophysics , 1(2004): 156-163.

[9] R. B. Herrmann. SH-wave generation by dislocation sources-a numerical study. Bulletin of the Seismological Society of America, 1(1979): 1-15. 